\title{
Repeated Single Level Lumbar Sympathetic Neurolysis using Alcohol in a Plantar Hyperhidrosis Patient: A 5-year Follow-up Case Report
}

\author{
Yeong-Gwan Jeon ${ }^{1}$, Young-Hyun Jin ${ }^{1}$, Hyun-Jae $\mathrm{Im}^{1}$, Young-Bok Lee ${ }^{{ }^{*}}$ \\ ${ }^{1}$ Department of Anesthesiology and Pain Medicine, Wonju College of Medicine, Yonsei University, 20 Ilsan-ro, Wonju 26426, \\ Ganwon-do, Republic of Korea \\ Email: ygjeon [AT] yonsei.ac.kr \\ ${ }^{1}$ Department of Anesthesiology and Pain Medicine, Wonju College of Medicine, Yonsei University, 20 Ilsan-ro, Wonju 26426, \\ Ganwon-do, Republic of Korea \\ Email: wlsdudgus92 [AT] gmail.com \\ ${ }^{1}$ Department of Anesthesiology and Pain Medicine, Wonju College of Medicine, Yonsei University, 20 Ilsan-ro, Wonju 26426, \\ Ganwon-do, Republic of Korea \\ Email: closecl [AT] naver.com \\ ${ }^{1}$ Department of Anesthesiology and Pain Medicine, Wonju College of Medicine, Yonsei University, 20 Ilsan-ro, Wonju 26426, \\ Ganwon-do, Republic of Korea \\ *Corresponding author's email: yblee [AT] yonsei.ac.kr
}

\begin{abstract}
----
Background: Plantar hyperhidrosis causes discomfort in daily life. However, there are few reports on sympathetic neurolysis in plantar hyperhidrosis.

Case: A 19-year-old woman complained of excessive sweating of both hands and feet. Using video-assisted thoracic surgery, the patient underwent bilateral T3 sympathectomy in the department of chest surgery. One year later, she visited pain clinic with with plantar hyperhidrosis and wanted to undergo sympathetic block. Lumbar sympathetic neurolysis was performed using $2.5 \mathrm{ml}$ of pure anhydrous alcohol at the $\mathbf{L} 4$ vertebral level. The symptoms improved after the procedure, and there were no side effects. The patient again underwent sympathetic neurolysis as the symptoms recur.

Conclusions: Repeated lumbar sympathetic neurolysis was performed in a patient with plantar hyperhidrosis, and its success was confirmed during 5 years of follow-up.
\end{abstract}

Keywords--- Chemical neurolysis; Lumbar sympathetic block; hyperhidrosis; Plantar hyperhidrosis.

\section{INTRODUCTION}

Hyperhidrosis interferes with daily life. It affects the patient's personal and professional life as well as mental health, thereby reducing the quality of life (QOL) [1]. Hyperhidrosis is caused by pathologic hyperactivity of the sympathetic system. It is difficult to control excessive sweating. Therefore, patient's daily and vocational activities are much limited; hence, most patients seek treatment [2]. Topical agents, iontophoresis, and anticholinergics are recommended as first-line treatments. In case of the medically intractable palmar hyperhidrosis, thoracoscopic sympathectomy can be performed next [3]. However, sympathectomy is rarely performed for the plantar hyperhidrosis.

Lumbar sympathetic neurolysis is used to treat severe ischemic rest pain or peripheral arterial disease of the lower extremities. Furthermore, it can be used for the treatment of complex regional pain syndrome, peripheral vasospasm, postherpetic neuralgia, phantom limb pain, and plantar hyperhidrosis [4].

Here, we present a successful treatment of plantar hyperhidrosis using lumbar sympathetic neurolysis.

\section{CASE REPORT}

The current report was approved by Institutional Review Board (CR321334).

A 19-year-old female patient visited the pain clinic with complaints of persistent sweating of both feet after thoracic 
sympathectomy. She had a history of thoracic sympathectomy 1 year ago and had no specific medical history. She wanted to undergo a sympathetic nerve block. We decided to perform left lumbar sympathetic neurolysis using alcohol at the L4 level. After explaining the effects and side effects of the procedure, informed consent was obtained, and it was confirmed that the patient did not have any specific allergies.

The patient was placed in the right lateral decubitus position with a pillow at the flank under non-invasive blood pressure, pulse oximeter, and electrocardiogram monitoring in the operating room. Under fluoroscopic guidance, the fourth lumbar vertebral body was confirmed in the anteroposterior (AP) view and rotated obliquely to confirm the needle entry point. After the skin was prepared using povidone-iodine, a skin wheal was made with $2 \%$ lidocaine at the needle entry point 7 $\mathrm{cm}$ lateral to the upper boundary of the spinous process up to L4. A 23 -gauge, 12-cm needle was inserted with the gunbarrel technique in oblique view. When the needle tip touched the lateral margin of the vertebral body, the needle was advanced in a sliding-like motion to the anterolateral border under lateral view. The location of the needle tip was confirmed at the lateral half of the pedicle in the AP view and at the anterior margin of vertebral body in the lateral view. For the test block, a mixture of $1.5 \mathrm{ml}$ of contrast dye (Hexosure ${ }^{\circledR}, 300 \mathrm{mg} \mathrm{I} / \mathrm{ml}$; Pharvis Korea, Seoul, Korea) and $1.5 \mathrm{ml}$ of $4 \%$ lidocaine was injected, and longitudinal spreads were confirmed; no intramuscular or intravascular injections were not observed (figure 1.).

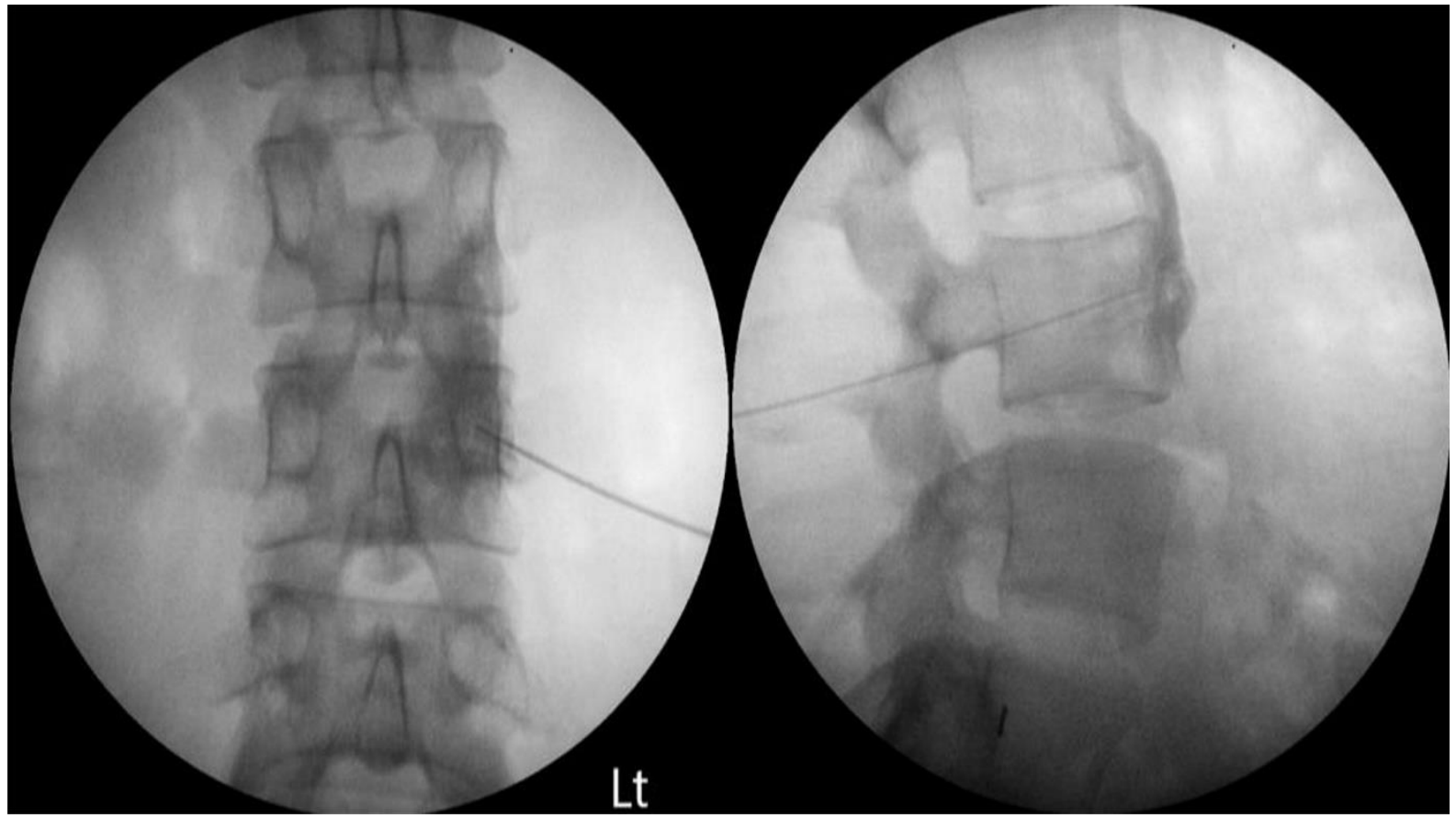

Figure 1 : The needle tip was positioned at lateral half of the pedicle in the anteroposterior view and at the anterior margin of the vertebral body in the lateral view. Contrast dye was distributed longitudinally without intramuscular or intravascular injections.

After the test block, the left foot became warm and dry, with no motor or sensory impairments in the left lower extremity. After 15 minutes, $2.5 \mathrm{ml}$ of $99 \%$ anhydrous alcohol was carefully injected. The patient did not complain of any discomfort during the injection and was then moved to the recovery room; there were no side effects. One month later, upon the patient's request, she underwent right lumbar sympathetic neurolysis on L4, and again, there were no side effects.

There was a recurrence of symptoms, and lumbar sympathetic neurolysis was performed three times on the right and two times on the left. The shortest period was 6 months (once) and 19-21 months (four times), with 21 months as the longest period. The average symptom improvement period was 17 months. The last block was administered on March 26, 2021, and both feet were in a satisfactory state without sweating at the most recent follow-up.

\section{DISCUSSION}

Hyperhidrosis is characterized by excessive sweating, and more than $90 \%$ of cases are caused by primary hyperhidrosis. Primary hyperhidrosis occurs mainly between 14 and 25 years, and most cases occur in the plantar or palmar regions (88.9\%) [5]. Topical $20 \%$ aluminum chloride is the first-line treatment for primary hyperhidrosis. Botulinum toxin A injection or iontophoresis can be used as a first- or second-line treatment. Oral anticholinergics can be used for severe hyperhidrosis, which does not respond to the above-mentioned treatments [6]. Oral anticholinergic drugs generally use glycopyrrolate, which is known to have a greater perspiration suppression effect and fewer side effects than other 
anticholinergic drugs [7]. In severe cases of palmar hyperhidrosis that do not respond to non-invasive therapy, thoracic sympathectomy is used [6]. However, in the case of severe plantar hyperhidrosis, sympathectomy is not recommended because of concerns about side effects such as compensatory sweating, heat intolerance, and the irreversible nature of the procedure [8]. Nevertheless, there are cases of effective treatment of plantar hyperhidrosis through endoscopic lumbar sympathectomy [9].

Lumbar sympathetic neurolysis is used to treat various lower-limb diseases. Chemical neurolysis is mainly performed using absolute alcohol and performed under fluoroscopic guidance at the L2 to L4 lumbar vertebral level. Single-level injection is generally performed at L3, and multilevel injection is performed to further spread longitudinally. In our case, we performed sympathetic neurolysis at L4, not for the whole lower extremity, but for plantar hyperhidrosis. A study reported that sympathetic neurolysis on the L4 or L4/5 disc segment is more effective than on the other segments for plantar hyperhidrosis [10]. However, a conflicting study showed that the success rate in L3 to L4 is higher than that in L4 [11]. Therefore, further research is needed to determine which level of sympathetic neurolysis is more effective.

Side effects include bleeding, nerve root injury, genitofemoral neuralgia, neuraxial injection, paralysis, and renal injury. Genitofemoral neuralgia is caused by injection into the psoas muscle [4]. Intramuscular injections occur more frequently when administered at multiple levels [12]. Unlike local anesthetics, which have a temporary blocking effect, neurolysis using alcohol can have a long-term or permanent effect; therefore, only small amounts are used to minimize complications. Research on the proper amount of alcohol is insufficient, but $3 \mathrm{ml}$ is used per needle in most cases [13,14]. However, complications often occur when injected at multiple levels.

Considering the young age and symptoms of the patient in our case, neurolysis was performed only at the L4 level to minimize side effects rather than maximize the treatment effect. In addition, $2.5 \mathrm{ml}$ of alcohol was used, and no complications were observed during or after the procedure. The procedure was performed on the left side at first and then on the right side 1 month later. Subsequently, it was performed on the side with symptoms. Even though only a small amount of alcohol was used on one side of the L4 level, the average symptom improvement period was 17 months. In our case, there was only one case of recurrence within 1 year, which may be due to incomplete neurolysis. In one study, hyperhidrosis recurred within 1 year in $37 \%$ of patients and between 13 and 18 months in 58\% of patients after lumbar sympathetic neurolysis [11]. In another study, 34\% of cases had recurrence within 2 years after lumbar sympathetic neurolysis, with $81 \%$ recurring within 1 year and $19 \%$ recurring within 1 year to 2 years [14]. Compared with these studies, we believe that the average symptom improvement period in our case was maintained for a long period. The patient's satisfaction was high, and until recently, she had undergone repeated treatments.

As a result, we confirmed satisfactory effects for a sufficient period of approximately 17 months by repeatedly (seven times) performing L4 lumbar sympathetic neurolysis using alcohol for plantar hyperhidrosis. No complications occurred during the procedures.

\section{REFERENCES}

1. Lenefsky M, PharmD, Rice ZP. Hyperhidrosis and Its Impact on Those Living With It. Am J Manag Care 2018; 24: S491-5.

2. Schick CH. Pathophysiology of Hyperhidrosis. Thorac Surg Clin 2016; 26: 389-93.

3. Benson RA, Palin R, Holt PJ, Loftus IM. Diagnosis and management of hyperhidrosis. BMJ 2013; 347 : f6800.

4. Zechlinski JJ, Hieb RA. Lumbar Sympathetic Neurolysis: How to and When to Use? Tech Vasc Interv Radiol 2016; 19: 163-8.

5. Nawrocki S, Cha J. The etiology, diagnosis, and management of hyperhidrosis: A comprehensive review: Etiology and clinical work-up. J Am Acad Dermatol 2019; 81: 657-66.

6. McConaghy JR, Fosselman D. Hyperhidrosis: Management Options. American Family Physician 2018; $97: 729-$ 34.

7. Lee HH, Kim DW, Kim DW, Kim C. Efficacy of glycopyrrolate in primary hyperhidrosis patients. Korean J Pain 2012; 25: 28-32.

8. Vlahovic TC. Plantar Hyperhidrosis: An Overview. Clin Podiatr Med Surg 2016; 33: 441-51.

9. Rieger R, Pedevilla S, Lausecker J. Quality of life after endoscopic lumbar sympathectomy for primary plantar hyperhidrosis. World J Surg 2015; 39: 905-11.

10. Kim HJ, Lee CO, Shin Y-S, Lee Y-W. Appropriate Block Level in Neurolytic Lumbar Sympathetic Ganglion Block. Korean J Pain 2001; 14: 199-206.

11. Kim WO, Yoon KB, Kil HK, Yoon DM. Chemical lumbar sympathetic block in the treatment of plantar hyperhidrosis: a study of 69 patients. Dermatol Surg 2008; 34: 1340-5. 
12. Hong JH, Oh MJ. Comparison of Multilevel with Single Level Injection during Lumbar Sympathetic Ganglion Block: Efficacy of Sympatholysis and Incidence of Psoas Muscle Injection. Korean J Pain 2010; 23: 131-6.

13. Chung Y-J, Choi JB, Lee Y-W. Radiofrequency Lumbar Sympatholysis Comparison with Neurolytic Alcohol Block. Korean J Pain 2004; 17: 42-6.

14. Han ST, Kim C, Han KR, Cho HW, Noh HJ. Lumbar Sympathetic Ganglion Block with Alcohol for Plantar Hyperhidrosis. Korean J Pain 2005; 18: 161-4. 